This item was submitted to Loughborough's Institutional Repository (https://dspace.lboro.ac.uk/) by the author and is made available under the following Creative Commons Licence conditions.

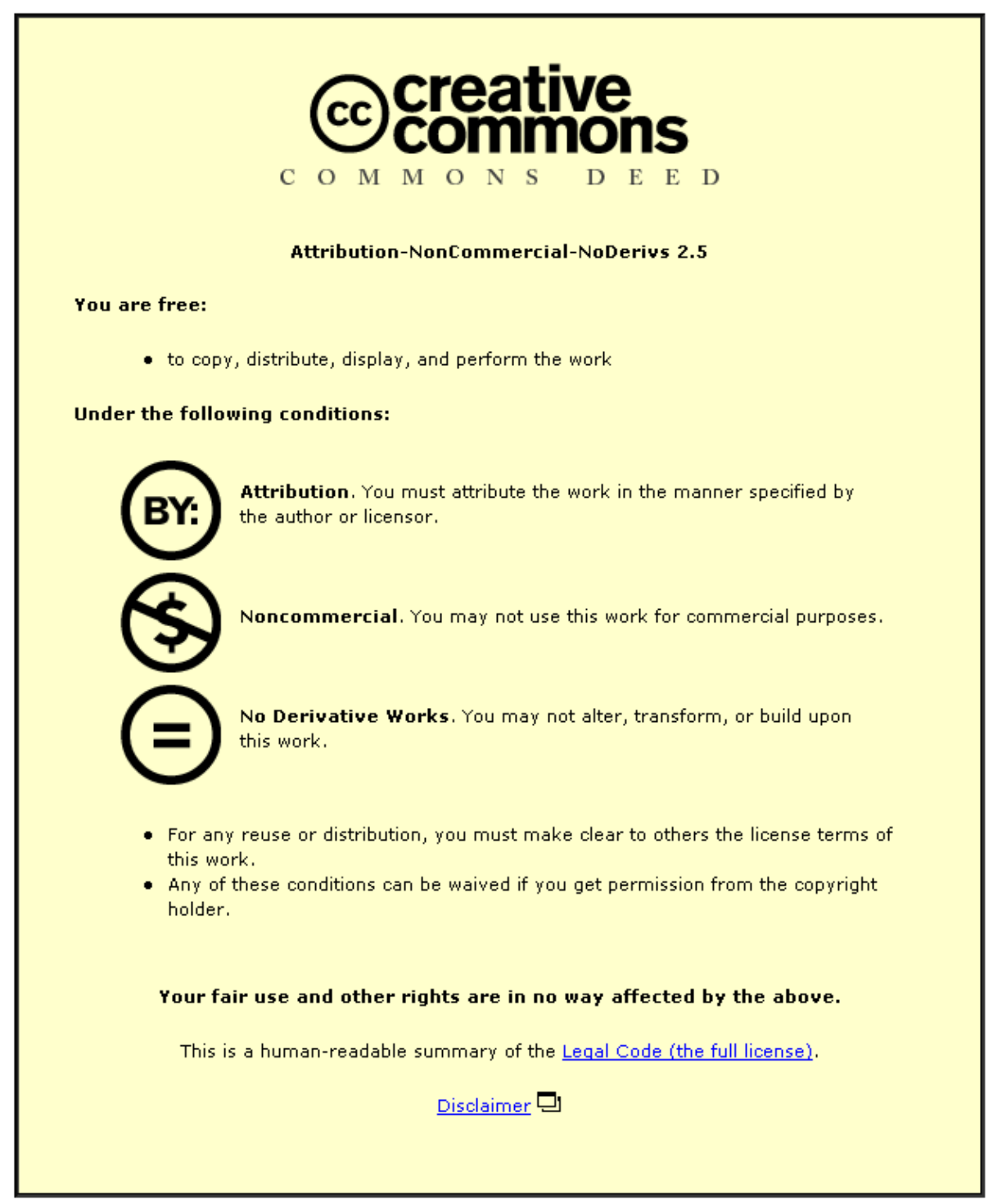

For the full text of this licence, please go to: http://creativecommons.org/licenses/by-nc-nd/2.5/ 


\title{
Twisting Double Somersault High Bar Dismounts
}

\author{
Maurice R. Yeadon \\ Department of Sports Science, Loughborough University, LE11 3TU, United Kingdom
}

\begin{abstract}
At the 1988 Seoul Olympic Games, four double somersaults dismounts with one twist and four double somersault dismounts with two twists were filmed using two $16 \mathrm{~mm}$ cameras during the men's horizontal bar competition. Contributions to tilt angles reached at the mid-twist position, determined using computer simulations based on modifications of the data obtained from film, were used as measures of the twisting potential of various techniques. The amount of tilt produced was greater when the total twist was greater and when the body was tucked rather than straight. The twisting techniques used varied with the timing of the twist within the two somersaults. Contact contributions were larger when there was more twist in the first somersault. When there was little or no twist in the first somersault, the major contribution came from aerial techniques that comprised mainly arm movements and asymmetrical hip movements in the flight phase.
\end{abstract}

\section{Introduction}

Dismounts from the high bar comprise single or multiple somersaults with or without twist. In nontwisting dismounts, the gymnast must leave the apparatus with the appropriate linear and somersaulting velocities and must then adjust body configuration during flight to obtain the correct somersault angle at landing. In twisting dismounts, in addition to meeting the above requirements, the gymnast must also initiate and control twisting to produce the required amount of twist prior to landing. Twisting techniques may be classified according to whether the twist starts during contact with the apparatus or is initiated during the aerial phase. This gives rise to the terms contact techniques and aerial techniques for the production of the twist.

For dismounts from the high bar, the gymnast may initiate twist prior to releasing the bar by twisting the trunk and lower body relative to the shoulders. This will induce a torque on the hands from the bar that results in the build-up of angular momentum about the longitudinal axis during the contact phase. One consequence of using contact twist in a somersault is that the longitudinal axis of the body moves out of the vertical plane in which it lies at takeoff (Yeadon, 1993b). After a complete somersault this tilt away from the vertical plane will disappear of its own accord, if no configurational changes are made. In the case of a one-and-a-half somersault dive with one-and-a-half twists, the body will tend to be tilted sideways at entry into water. In such a case there may be problems in completing the dive successfully since there is no ideal solution in which the body lies in the vertical plane and the twist velocity is zero (Yeadon, 1993b). In the case of high bar single and double somersault dismounts with twist, the total somersault rotation in flight will be about three-quarters of a revolution or one and three-quarter revolutions, since the body is close to the horizontal at release. It is possible, therefore, that too much contact twist may lead to problems at landing for high bar dismounts. If this is so, it may be expected that gymnasts will use aerial techniques in preference to contact techniques.

Aerial twist may be produced by counter-rotation using a hula-hoop motion of the hips, but this technique requires one cycle of hip movement for each half twist of the body (Yeadon, 1993c). The gymnast may initiate a sustained aerial twist during a plain somersault by tilting the longitudinal axis of the body away from the plane perpendicular to the angular momentum vector (Batterman, 1974). In theory this may be accomplished using asymmetrical movements of the arms, chest, or hips (Yeadon, 1993c).

While there are a number of viable twisting techniques that gymnasts can use, information on which techniques are actually employed is limited. Biesterfeldt (1974) believed that there was little tilt of 
the body away from the vertical somersault plane and that the twist was produced by counter-rotation during the aerial phase, in the same way that a cat twists when dropped from an inverted position. In contrast, Gluck (1979) presented sequences of single somersault dismounts from the high bar, with one, two, and three twists, which showed that the tilt away from the vertical plane after a half somersault increased with the number of twists. This observation is consistent with the use of contact twist, or of aerial twist produced by tilt (Hopper, 1973), but is not consistent with the use of the counter-rotation aerial techniques proposed by Biesterfeldt.

Al Haroun (1980) filmed six gymnasts performing full twisting dismounts from the rings. Three gymnasts started the twist before releasing first one ring and then the other, while the remaining three released the two rings simultaneously and appeared to initiate the twist after release. Conclusions drawn from such a descriptive study should be viewed with some caution, since it can only be suggested that a particular technique was used. A more justifiable result was obtained by Van Gheluwe (1981), who used a six-segment computer simulation model to evaluate filmed performances of three full twisting somersaults on a trampoline. By using simulations based on modifications of the film data, the author showed that asymmetrical arm movements during the aerial phase produced the twist.

A method of partitioning the twist contributions was developed by Yeadon (1993d), who used the angle of tilt as a measure of the amount of twisting. Using an 11-segement simulation model, Yeadon, Lee and Kerwin (1990b) showed that for eight single somersaults dismounts from the high bar with one twist, the majority of the tilt arose from asymmetrical movements during the aerial phase. For six double somersault dismounts from the rings with one twist in the second somersault, the majority of the tilt was a result of asymmetrical arm movements in the aerial phase (Yeadon, 1994).

Many dismounts from the high bar take the form of double somersaults with twist. There is a great variety of such movements since the release may be from either a forward or backward giant circle, the number of twists can vary, and the timing of the twist within the two somersaults can vary. In trampolining, twisting double somersaults are named on the basis of the amount of twist in each somersault. The twisting techniques that gymnasts use may depend upon the type of dismount and on their level of expertise. In this study, a variety of backward double somersault dismounts with twist, as performed by Olympic competitors, are analyzed to determine the contributions of contact and aerial techniques.

\section{Methods}

\section{Data Collection}

The high bar exercise of the Men's Artistic Gymnastics all-around and individual competitions at the 1988 Seoul Olympic Games were filmed using two Photosonics 1 PL 16 mm cine cameras. The cameras were placed at the rear of the tiered seating areas to obtain front and side views of the dismounts. The camera locations and two points on one of the vertical supports of the high bar were surveyed for later use in camera calibration. The cameras were operated at nominal framing rates of $70 \mathrm{fps}$ and provided around 90 frames for the aerial phase of each dismount. Prior to the competition, 35 mm slides were taken of the seven members of the Canadian team to provide front and side views from which anthropometric data could be obtained on Olympic competitors for the purpose of calculating segmental inertia parameters.

\section{Data Processing}

All the backward double somersault dismounts with one and two twists were viewed, and the phasing of the twist within the somersaults was noted for each dismount. Four single twisting and four double twisting dismounts were selected to provide a variety of timings for the twist. Where there was more than one dismount with the same phasing of the twist within the two somersaults, a choice was made first concerning the quality of the movement and second on the degree of extension. This resulted in four straight single twisting dismounts and four double twisting dismounts of which one was straight and three were tucked. The dismounts were named according to the amount of twist in each somersault.

The front and side views of the aerial phase of each dismount were digitized. Each corner of the projected film frame was digitized four times, and their mean coordinate values were taken to determine 
the center of the film frame. Each image of the upper and lower points on one of the vertical supports of the high bar was digitized 16 times, and mean coordinate values were obtained. From the surveyed three-dimensional locations of the front camera, side camera, and two points on the high bar support together with the digitized data, the cameras were calibrated according to the method of Yeadon (1989).

In each film frame of the aerial phase, the wrist, elbow, shoulder, hip, knee, and ankle centers were digitized, providing 12 landmarks in total. The head was assumed to be in normal alignment and the ankles were assumed to be plantarflexed. The film from each camera was digitized twice to facilitate error correction and to produce error estimates for use in fitting quintic splines to the data. A combination of global and local error estimates was used as described in Yeadon (1990a) for curve-fitting the coordinate data using the quintic spline of Wood and Jennings (1979). These mathematical fits to the digitized data permitted the estimation of data values at times in between those of the film frames. The digitized data sets from the two cameras were synchronized by determining rays from each camera to an unweighted mean position of the 12 digitized points for the first three and last three frames of each dismount from each camera. The method described in Yeadon (1989) was used to determine the times of the second and penultimate frames from one camera in terms of the time scale of the other camera. The quintic spline fits to the displacement data were then used to obtain interpolated values to produce synchronous data sets.

The body was modelled using four arm segments, four leg segments, and three torso segments. The orientation of the body was defined by angles corresponding to somersault, apparent tilt, and twist, where somersault is the rotation about a horizontal axis, twist is the rotation about the longitudinal axis, and apparent tilt is the angle between the longitudinal axis and the vertical somersault plane (Figure ??). Body configuration was specified using 14 angles to describe the relative orientations of the body segments. These 14 angles comprised angles of flexion, abduction, and rotation at each shoulder joint; flexion angles at each elbow; tilt and torsion of the chest relative to the abdomen; one abduction angle for both thighs; one flexion angle for both knee joints; one flexion angle for the hip joints and the joint at the junction of the pelvis and abdomen; and one "hula" angle indicating whether flexion was forwards, backwards or sideways (Yeadon, 1990c). The three-dimensional coordinates of the digitized joint centers were reconstructed from the digitized data (Yeadon, 1989), and the time histories of the orientation and configuration angles were determined (Yeadon, 1990a).

Anthropometric measurements were obtained by digitizing the front and side views of the $35 \mathrm{~mm}$ film of the seven Canadian team members that had been taken prior to the competition. Segmental masses, mass center locations, link lengths, and moments of inertia were determined (Yeadon, 1990b; Yeadon, Challis, \& Draper, 1992).

\section{Data Analysis}

An 11-segment model was used to determine the angular momentum vector during each dismount for each of the seven sets of segmental inertia parameters using the time histories of the 17 orientation angles (Yeadon, 1990c). The mean values of the calculated angular momentum components and the time histories of the 14 configurational angles, together with the initial values of the three orientation angles and the segmental inertia parameters, were used as input to an 11-segment simulation model (Yeadon, Atha, \& Hales, 1990a). The segmental inertia set for each competitor was selected to be that which produced the closest agreement between the twist values obtained from simulation and film.

The simulation model was used to determine the direction of the longitudinal axis corresponding to minimum moment of inertia and to calculate the angle of tilt between longitudinal axis and the invariable plane perpendicular to the angular momentum vector. This angle of tilt is a kinetic variable in the sense that it describes an aspect of the mechanics of the system. It should be distinguished from the angle of apparent tilt, which is a kinematic variable describing orientation relative to the vertical (Yeadon, 1993b).

The relationship between the twist rate $p$, the tilt angle $\theta$, and the somersault rate $\Omega$ for an axially symmetric rigid body with principal moments of inertia $A, A$, and $C$ (where $A>C$ ) is given in Yeadon (1993a) as:

$$
p=\Omega\left(\frac{A}{C}-1\right) \sin \theta
$$




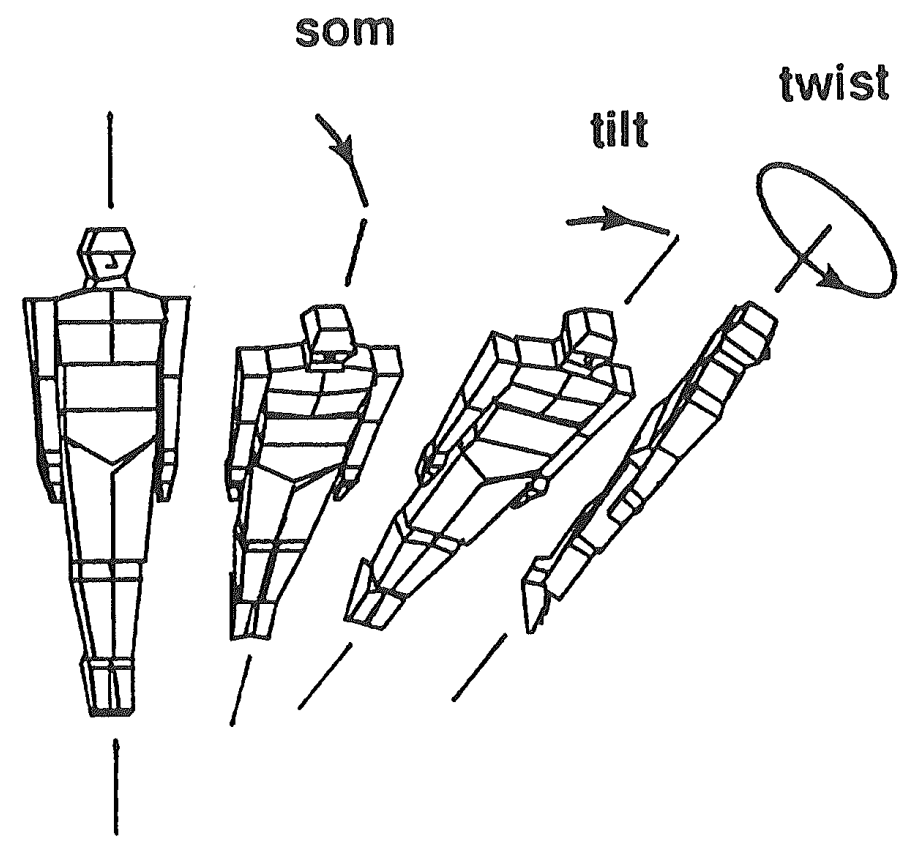

Figure 1: Angles of somersault, apparent tilt, and twist.

Thus, the angle of tilt between the longitudinal axis of the body and the invariable plane perpendicular to the angular momentum vector may be used to determine the number of twists per somersault for a given body configuration, providing that the two large principal moments of inertia are approximately equal. Since the angle of tilt is approximately proportional to the twist rate for a given body configuration, it may therefore be used as a measure of the twisting potential present, independent of the actual configuration adopted. In other words, there is a maximum twist rate associated with a particular tilt angle corresponding to a straight body configuration with arms and legs adducted. The actual twist angle will, of course, depend upon the body configuration used and the time at which the twist is initiated. The advantage of using the tilt angle (or potential twist rate) as a measure is that the contributions of various techniques are additive, whereas this is not the case if the twist angle is used. Twisting techniques are defined as contact or aerial techniques, depending on whether the twist is initiated during contact with the apparatus. The subsequent increase in tilt angle during the dismount gives the contribution of aerial twisting techniques to the total tilt produced.

In order to partition the aerial contribution to the tilt angle into a symmetry contribution and contributions associated with left-right asymmetries of arms, chest, and hips, the configurational angles obtained from film were modified: that is, asymmetries of the arms, chest, and hips relative to the sagittal plane were systematically removed. Arm movement was made symmetrical by having the left arm mirror the recorded movement of the right arm and vice-versa; chest torsion relative to the abdomen was eliminated; and lateral flexion was removed from the movement of the abdomen and legs relative to the pelvis by only permitting flexion parallel to the sagittal plane. The systematic removal of arm, chest, and hip asymmetries provided 12 configuration sequences ranging from the original movement to a sequence with complete left-right configurational symmetry. The tilt angle produced at the time corresponding to the mid-twist position in the simulation of the original movement was determined from simulations based upon the modified configurations. Since arm asymmetries were removed in two ways, the tilt values were averaged in these simulations to provide a total of eight tilt values for the modified simulations. The tilt contribution due to symmetry was calculated as the amount that the tilt angle rose above the initial values in a simulation in which left-right configurational symmetry was maintained.

Tilt associated with asymmetrical arm movement could be calculated as the difference between the tilt values for the simulations of complete symmetry and of symmetrical chest and hips. It could also be calculated as the difference between the tilt values for the simulation corresponding to arm symmetry and the original configuration. The average of the tilt values obtained in these two ways was taken as the tilt 
contribution from asymmetrical arm movement. The other contributions to tilt angle were calculated in a similar way as described by Yeadon (1993d). The total of the tilt contributions was compared with the actual tilt angle produced in the unmodified simulation as a test of the additivity of the contributions.

\section{Results and Discussion}

Since the interpolated information sets from the two cameras were synchronised using the displacement data, the accuracy of the synchronization depends upon the accuracy of the displacement data. Reliability of the synchronization of the two cameras views was estimates by taking the absolute difference between the values obtained from the two digitizations. These error estimates of the synchronization had a mean value of $0.002 \mathrm{~s}$. This should be regarded as an underestimate of the synchronization error since there will also be some systematic errors associated with error in the measured camera locations.

Mean error estimates of the reliability of repeated digitization were $0.010 \mathrm{~m}$ and $0.012 \mathrm{~m}$ for the eight dismounts. The difference between the values of the orientation angles obtained from simulation and film were evaluated at mid-flight time. Mean deviations were $0.02 \mathrm{rev}$ for somersaults, $2.0^{\circ}$ for apparent tilt, and $0.05 \mathrm{rev}$ for twist. Figure ??, which presents a typical comparison of film and simulation sequences for Competitor 132, shows that the differences between the two sequences increase as the simulation progresses. This is to be expected, since the errors will be cumulative due to the process of numerical integration inherent in a simulation.

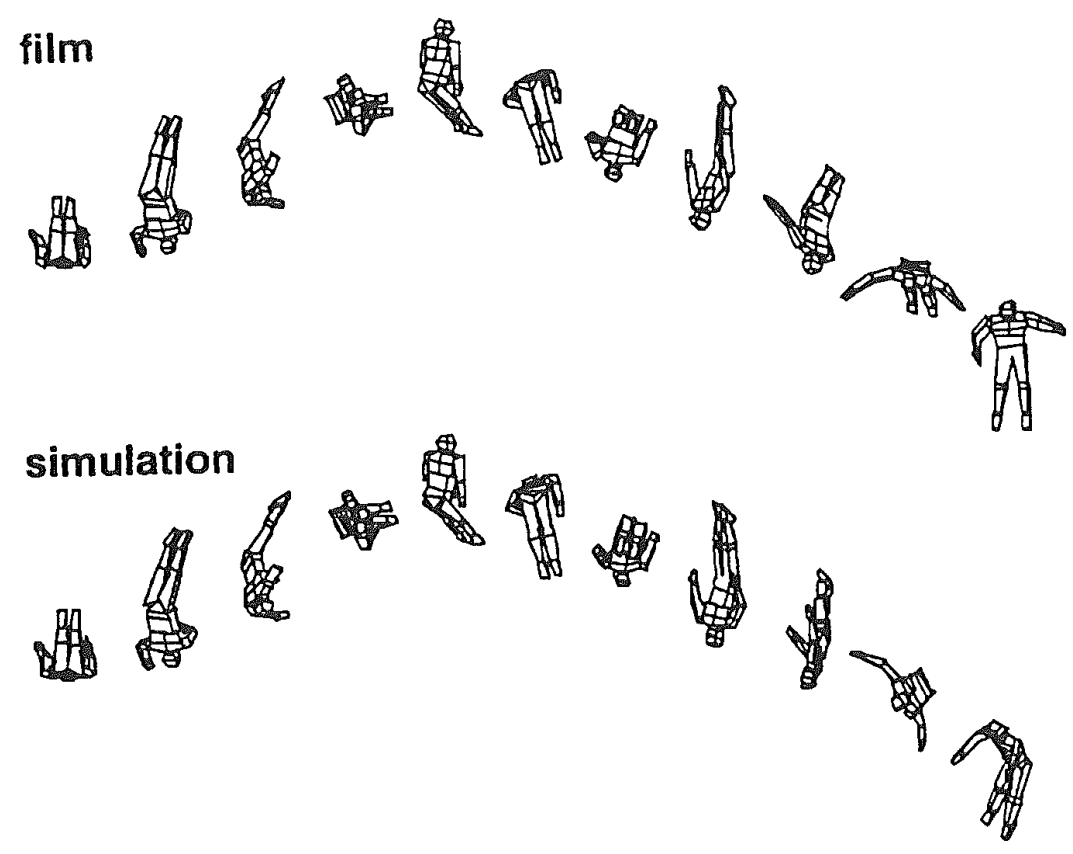

Figure 2: Comparison of film and simulation for Competitor 132.

Tilt contributions to the production of twist were evaluated for the time at which the original simulation reached the mid-twist position (Table ??). The first four dismounts listed are backward doubles with one twist while the next four are backward doubles with two twists (Figure ?? and ??). One might expect the gymnast to use the straight position when the number of twists is greater, since the moment of inertia about the longitudinal axis is small when the body is extended. In fact, in all of the single twisting dismounts the gymnasts used the straight position (Table ??). Although the tucked position requires greater tilt for two twists compared to the straight body position, it requires less angular momentum for the two somersaults. It appears that in this trade-off between difficulty of twist and somersault, the somersault is dominant since most gymnasts find the double twisting double somersault harder to perform in the straight position. Since the dismounts were selected on the basis of an extended body position whenever available, the inclusion of three tucked double twisting double somersault dismounts 
indicates the difficulty of performing these dismounts in the straight position.

Table 1: Contributions (in Degrees) to the Production of Tilt

\begin{tabular}{llcccccccc}
\hline Dismount & Shape & Competitor & Contact & Symmetry & Arms & Chest & Hips & Total & Actual \\
\hline 1.0-in 0.0-out & Straight & 169 & 4 & 5 & 2 & 0 & -2 & 8 & 8 \\
0.5-in 0.5-out & Straight & 167 & -1 & -2 & 3 & 1 & 6 & 8 & 8 \\
0.5-in 0.5-out & Straight & 132 & 3 & -1 & 6 & 0 & 1 & 9 & 9 \\
0.0-in 1.0-out & Straight & 196 & 2 & 3 & 2 & 2 & -1 & 8 & 8 \\
Mean & & 2 & 1 & 3 & 1 & 1 & 8 & 8 \\
& & & & & & & & & \\
1.5-in 0.5-out & Tucked & 130 & 13 & 1 & 1 & 3 & 0 & 17 & 17 \\
1.0-in 1.0-out & Tucked & 153 & 10 & 0 & 2 & 2 & 7 & 21 & 21 \\
1.0-in 1.0-out & Straight & 200 & -1 & -2 & 8 & 1 & 5 & 10 & 10 \\
0.5-in 1.5-out & Tucked & 179 & 7 & 5 & -2 & 0 & -1 & 9 & 9 \\
Mean & & 7 & 1 & 2 & 1 & 3 & 14 & 14 \\
Overall mean & & & 5 & 1 & 3 & 1 & 2 & 11 & 11 \\
\hline
\end{tabular}

Note. Contributions have been rounded to the nearest degree. Chest contributions arise from torsion of the chest relative to the abdomen. Hip contributions arise from the lateral flexion of the abdomen and legs relative to the pelvis.

Table ?? presents the contributions made to the tilt angle at the mid-twist position in each of the eight dismounts. The contact contribution is the initial tilt angle between the longitudinal axis and the invariable plane perpendicular to the angular momentum vector evaluated at the moment of release. The symmetry contribution indicates how much the tilt angle increases from its initial value during a simulation that enforces symmetry of the arms, chest, and hips about the sagittal plane. The contributions listed under arms, chest, and hips arise from asymmetrical arm movements, torsion of the chest, and lateral flexion at the hips.

For each of the eight dismounts analyzed, the deviations between the sum of the tilt contributions (as listed under Total) and the actual tilt value obtained from the unmodified simulation had a mean value of $0.3^{\circ}$ and a maximum value of $0.4^{\circ}$. This is reflected in Table ??, where the total and actual values are identical for all dismounts since the values have been rounded to the nearest degree.

All four angles of actual tilt $\left(9.3^{\circ}\right.$ to $\left.21.1^{\circ}\right)$ for the double twisting dismounts were greater than the four tilt angles $\left(7.5^{\circ}\right.$ to $\left.8.5^{\circ}\right)$ for the full twisting double somersaults. It is to be expected that the tilt angle will be greater when there is more twist since the tilt angle is proportional to the twist in the case of an axially symmetric rigid body. The double twisting dismounts for which the body was tucked throughout the aerial phase (Competitors 130 and 153) had tilt angles around twice that of the double twisting dismounts for which the body was straight for the majority of the aerial phase (Competitors 200 and 179). This is to be expected, since the twist rate will be lower for a given tilt angle when the body is tucked rather than straight.

In the dismounts where twist occurred early in the first somersault (Competitors 169, 130, 153, 179), there was a large contact contribution. In the remaining dismounts, the twist started late in the first somersault and the contact contributions were small. This is also to be expected, since the use of contact twist will result in the gymnast twisting at release. As the amount of twist in the first somersault of the 


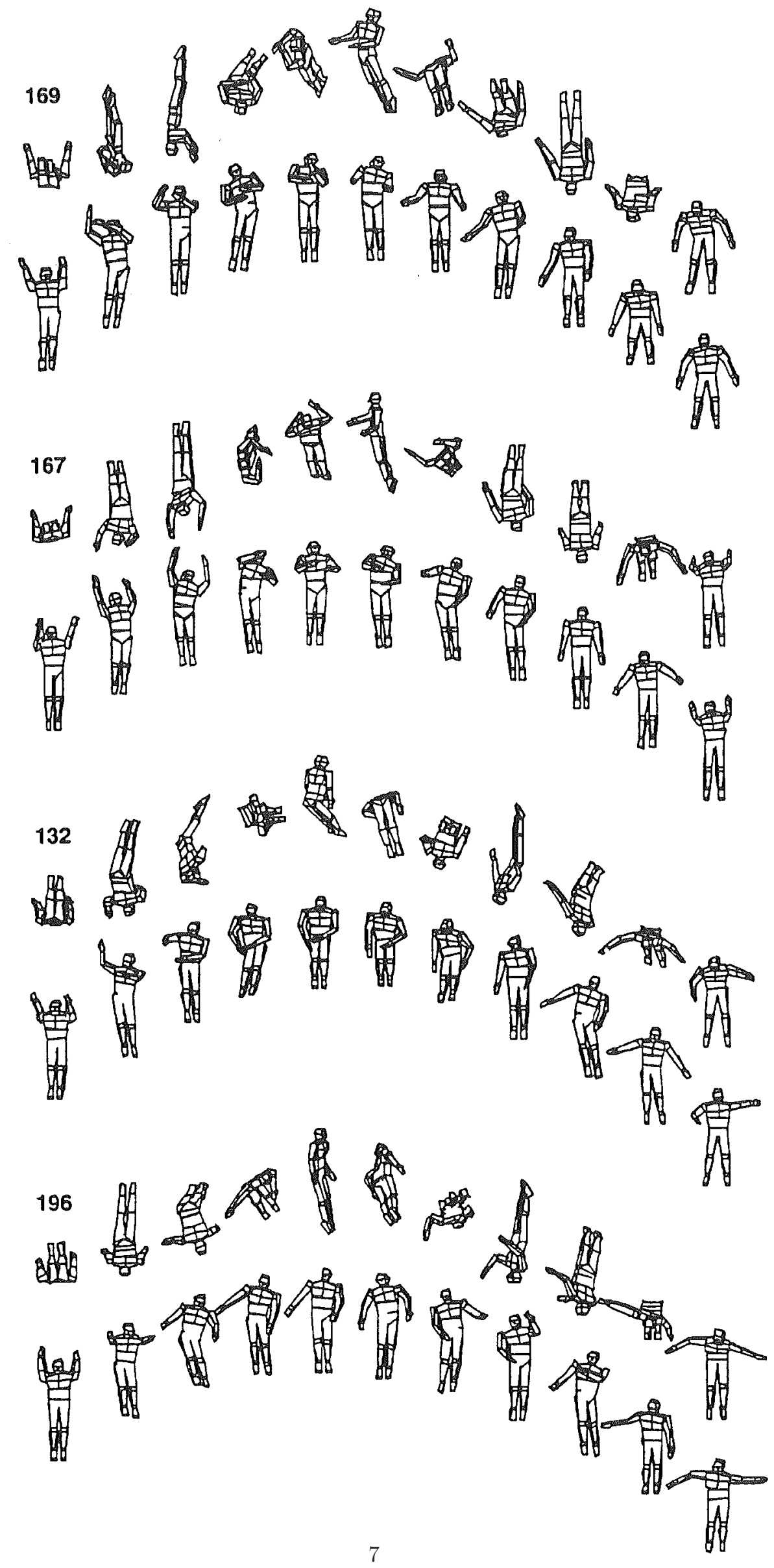




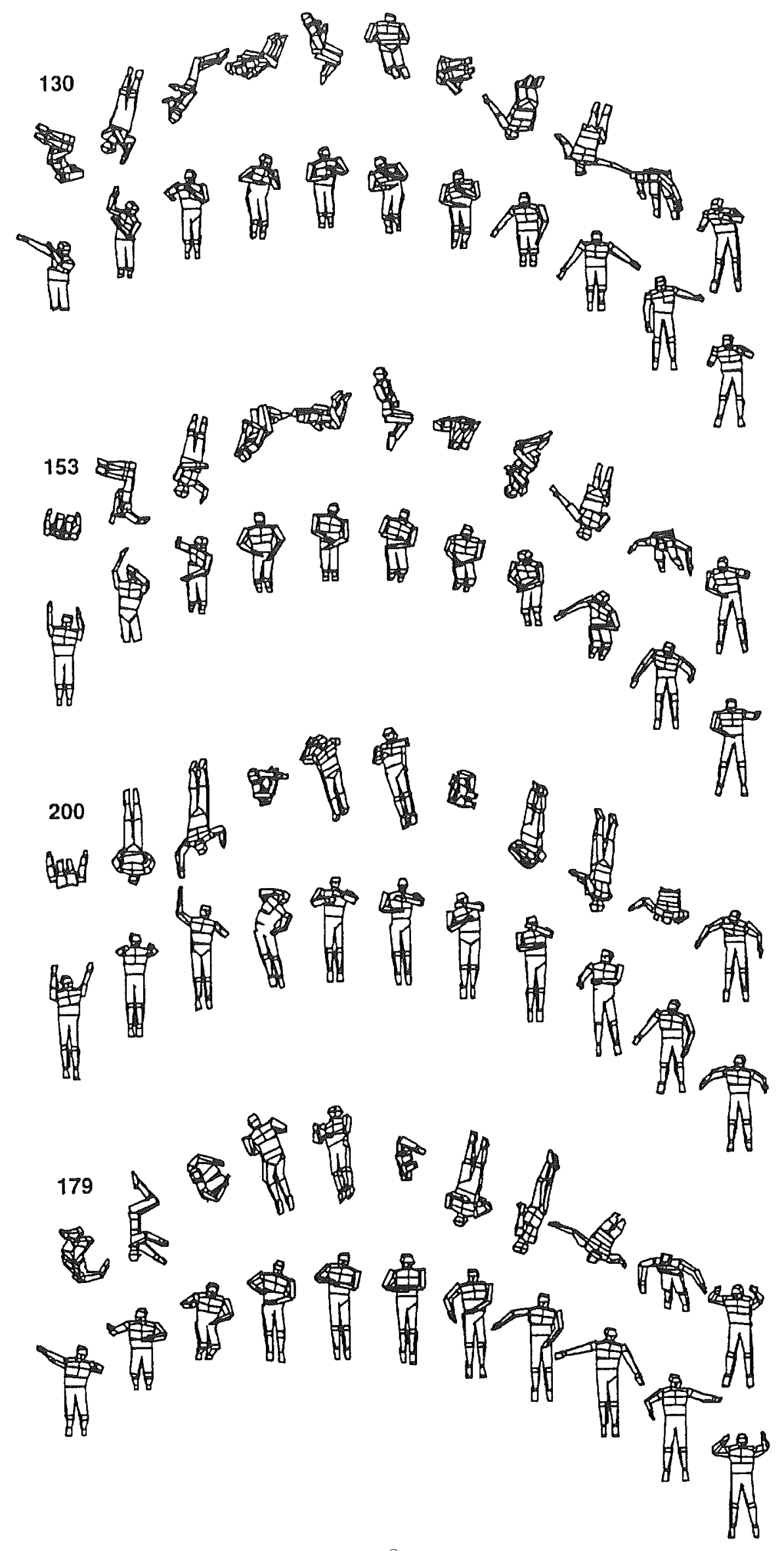

8

Figure 4: Graphics sequences of the four dismounts with double twist. Beneath each dismount sequence is a sequence showing the body configuration used. 
movement increased, the contact tilt at release also increased (Figure ??). The contact tilt and twist in the first somersault were significantly correlated $(\mathrm{r}=0.87, \mathrm{p}<0.001)$. The mean absolute deviation of the tilt values from the regression line $\mathrm{y}=6.4 \mathrm{x}$ through the origin was $2.7^{\circ}$. The two outliers with negative angles of contact tilt in Figure ?? correspond to the dismounts of Competitors 167 and 200, in which there was very little twist in the early part of the first somersault (Figures ?? and ??). While a substantial contact contribution will result in substantial twist in the first somersault, the reverse is not necessarily true.

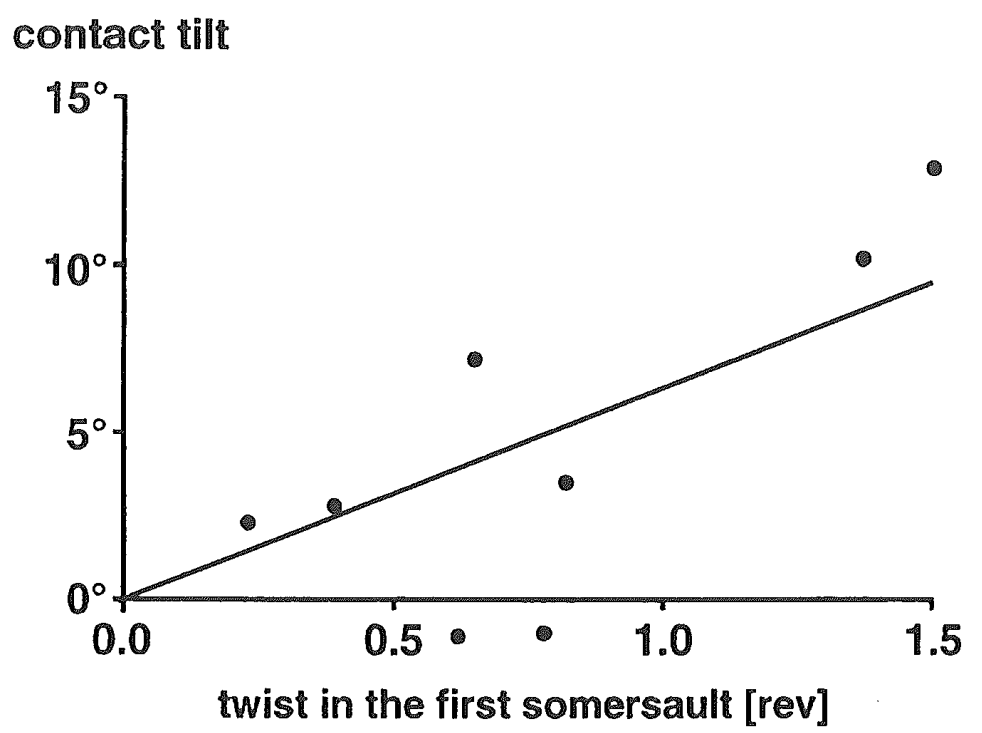

Figure 5: Contact tilt as a function of the twist in the first somersault.

In the first quarter somersault of the single twisting dismounts, twist is evident in the graphics sequences of Competitors 169 and 132 but is not evident for Competitors 167 and 196 (Figure??). For the double twisting dismounts, substantial twist is visible for Competitors 130, 153, and 179 but is not noticeable for Competitor 200 (Figure ??). Since each competitor twisted to the left and initially somersaulted backwards, the tilt was always in the same direction (Figures ?? and ??).

The techniques used for the dismounts were varied. Competitors 169, 132, 130, 153, and 179 had substantial contact contributions to the tilt. These initial tilt angles are not evident from the graphics sequences since tilt is measured from the plane perpendicular to the angular momentum vector and the presence of a twist angular velocity at release results in an angular momentum component about the longitudinal axis. The twist in the early part of the dismount is visible as noted above and indicates a contact contribution. Competitors 169, 196, and 179 had substantial symmetry contributions. Gymnasts can provide symmetry contributions to the tilt angle by maintaining a wide arm configuration so that rigid body nutation allows the tilt angle to increase until the quarter twist position is reached (Yeadon, 1993e). Competitor 196 exhibited such an arm configuration in the third graphic of the lower sequence in Figure ??. Another method of obtaining a symmetry contribution is to lower the arms in the sagittal plane at around the quarter twist position. This produces a counter-rotation of the body, which increases the tilt angle. Competitor 169 showed such an arm movement (Figure ??). Competitor 179 exhibited aspects of both arm symmetry techniques (Figure ??).

Competitors 132 and 200 displayed asymmetrical arm movements, raising the right arm relative to the left arm, which resulted in tilt contributions that accounted for the majority of the total tilt produced. While Competitors 169 and 130 also made asymmetrical arm movements early in the flight phase, the body had already twisted as a result of the contact contribution so that little realignment of the body occurred about the tilt axis. Although none of the chest torsion contributions to the tilt was particularly high, all contributions were positive. Large lateral hip flexion contributions were obtained by Competitors 167, 153 and 200. The lateral flexion of abdomen and legs relative to the pelvis was particularly evident for Competitor 200 in the fourth graphic of the lower configuration sequence in Figure ??. Flexion at the left hip accentuated the raising of the right arm and lowering of the left and 
produced tilt in the same direction as the tilt produced by the arms, so that the principal longitudinal axis was aligned more with the legs than with the trunk.

The twisting techniques used varied with the timing of the twist within the two somersaults. In general, contact contributions were large when there was substantial twist in the first somersault, whereas contact contributions were small when there was little or no twist in the first somersault. Aerial contributions were primarily a result of arm movements and lateral flexion at the hips during the flight phase. Since the techniques used varied with the timing of the twist within the two somersaults, investigators who analyze performances of just one twisting somersault skill will be in a better position to isolate the techniques used. Such information together with the results of hypothetical simulations (Yeadon, 1993b, 1993c) should enable coaches to develop progressions so that gymnasts can learn twisting skills more quickly and more safely. The present study is a first step in realizing this aim.

\section{References}

Al-Haroun, M.R. (1980). Three-dimensional cinematographic analysis of selected full twisting movements in gymnastics. Unpublished doctoral dissertation, Indiana University.

Batterman, C. (1974). The techniques of springboard diving ( $3^{\text {rd }}$ ed.) Cambridge, MA: MIT Press.

Biesterfeldt, H.J. (1974). Twisting mechanics II. Gymnast, 16(6/7), 46-47.

Gluck, M. (1979). So you want to twist: Part I. Ontario Gymnast, 31, 14-20.

Hopper, B.J. (1973). The Mechanics of human movement. London: Crosby Lockwood Staples.

Van Gheluwe, B. (1981). A biomechanical simulation model for airborne twist in backward somersaults. Journal of Human Movement Studies, 7, 1-22.

Wood, G.A. \& Jennings, L.S. (1979). On the use of spline functions for data smoothing. Journal of Biomechanics, 12, 477-479.

Yeadon, M.R. (1989). A method for obtaining three-dimensional data on ski jumping using pan and tilt cameras. International Journal of Sport Biomechanics, 5, 238-247.

Yeadon, M.R. (1990a). The simulation of aerial movement - I: The determination of orientation angles from film data. Journal of Biomechanics, 23, 59-66.

Yeadon, M.R. (1990b). The simulation of aerial movement - II: A mathematical inertia model of the human body. Journal of Biomechanics, 23, 67-74.

Yeadon, M.R. (1990c). The simulation of aerial movement - III: The determination of the angular momentum of the human body. Journal of Biomechanics, 23, 75-83.

Yeadon, M.R. (1993a). The biomechanics of twisting somersaults: Part I. Rigid body motions. Journal of Sports Sciences, 11, 187-198.

Yeadon, M.R. (1993b). The biomechanics of twisting somersaults: Part II. Contact twist. Journal of Sports Sciences, 11, 199-208.

Yeadon, M.R. (1993c). The biomechanics of twisting somersaults: Part III. Aerial twist. Journal of Sports Sciences, 11, 209-218.

Yeadon, M.R. (1993d). The biomechanics of twisting somersaults: Part IV. Partitioning performance using the tilt angle. Journal of Sports Sciences, 11, 219-225. 
Yeadon, M.R. (1993e). Utilising nutation in twisting somersaults. In Proceedings of the Fourth International Symposium on Computer Simulation in Biomechanics (BMS3-16, 17). Paris: Ecole Nationale Superiere d'Arts et Metiers.

Yeadon, M.R. (1994). Twisting techniques used in dismounts from the rings. Journal of Applied Biomechanics, 10, 178-188.

Yeadon, M.R., Atha, J., \& Hales ,F.D. (1990a). The simulation of aerial movement - IV: A computer simulation model. Journal of Biomechanics, 23, 85-89.

Yeadon, M.R., Challis, J.H., Draper, J.A. (1992d). Measurement of body segment parameters. Journal of Sports Sciences, 10, 589-590.

Yeadon, M.R., Lee, S., \& Kerwin, D.G. (1990b). Twisting techniques used in high bar dismounts. International Journal of Sport Biomechanics, 6, 139-146.

\section{Acknowledgment}

The assistance of Dr. Sung-Cheol Lee and the support provided by the International Olympic Committee Medical Commission and the Sport Canada Applied Research Programme are gratefully acknowledged. 